\title{
Transporte público e infraestructura urbana, causas de exclusión social
}

\section{Public transport and urban infrastructure, causes for social exclusion in old age}

Jennifer Aholibama Sánchez-Vázquez*

Ovidio Arturo González-Gómez*

Recibido: diciembre 01 de 2020.

Aceptado: mayo 03 de 2021.

\begin{abstract}
Resumen
Entre más avanza la edad de las personas, su inclusión a la sociedad y al entorno disminuye, debido a la pérdida de sus capacidades físicas, por lo que muchas personas de la tercera edad optan por resguardarse en sus hogares y/o atender sus necesidades en proximidad, así como adoptar al transporte privado como única opción para su movilidad y/o ser dependientes de un familiar. En este artículo se analiza si las condiciones que ofrece el transporte público y la infraestructura urbana son las causas principales por las que adultos mayores restringen su movilidad en la cabecera municipal de Santiago de Querétaro, México, mediante la aplicación de encuestas domiciliadas, con el fin de ofrecer elementos para renovar los procesos de diseño y evaluación del espacio público. Se exploran las características y las necesidades de los adultos mayores, así como las dificultades que enfrentan y sus requerimientos para desplazarse por el entorno público, pues la mayoría de las y los encuestados dicen sentirse excluidos por no ser tomados en cuenta.
\end{abstract}

Palabras clave: inclusión, Querétaro, movilidad, tercera edad, excluidos.

\begin{abstract}
As the age of the people advances, their inclusion in society and the environment decreases, due to the loss of their physical capacities, so many seniors choose to shelter in their homes and attend to their needs in proximity, as well as adopt private transport as the only option for their mobility and or be dependent of a relative. This paper analyzes whether the conditions offered by public transport and urban infrastructure are the main causes why older adults restrict their mobility in Santiago, Queretaro, through the application of domiciled surveys, and thereby propose elements to renew the processes of design and evaluation of public space, the characteristics and needs of the elderly are explored, as well as the difficulties they face and their requirements to move around the public environment, since most of the respondents say they feel excluded because they are not taken into account. Keywords: inclusion, Queretaro, mobility, old people, excluded.
\end{abstract}

Keywords: inclusion, Queretaro, mobility, old people, excluded.

\footnotetext{
* Universidad Autónoma de Querétaro, México. Correos electrónicos: jsanchez403@alumnos.uaq.mx, ovidio@uaq.mx
} 


\section{Introducción}

La población de personas de la tercera edad aumenta cada día a un ritmo acelerado; Osorio (2006) proyecta que la población de 60 años y más se triplicará entre los años 2000 y 2050 , "por lo que podemos decir que la población deja de crecer, ahora más bien envejece" (Zamorano et al., 2012). Por primera vez habrá más adultos mayores que niños (Morfi Samper, 2010); por lo tanto, la población del adulto mayor representará un alto porcentaje dentro de la población de cada país; por eso, se debe prestar más atención a todo lo que actualmente rodea al envejecimiento.

México, a lo largo de la última década, ha experimentado un intenso proceso de urbanización y una rápida transición demográfica hacia el envejecimiento, pues $10.5 \%$ de la población tiene 60 y más años, es decir, son personas de la tercera edad (INEGI, 2015).

El envejecimiento es un proceso de deterioro, en el cual se suman todos los cambios ocurridos con el tiempo en un organismo y que conducen a alteraciones fundamentales que, lamentablemente, provocan una pérdida progresiva de capacidad de adaptación. Además, las transformaciones ocurridas en los últimos años limitan la adaptabilidad en relación con su medio, pues el envejecimiento implica modificaciones graduales motoras y sensoriales y, por ende, una readaptación de las actividades de la vida cotidiana; se desarrollan desde tres variables: biológicas, psíquicas y sociales (Martínez Sellarés, 2012).

El reporte nacional de movilidad urbana en México 2014-2015 afirma que las personas de edad avanzada se ven obstaculizadas para trasladarse, ya sea como pasajeros o peatones, sufriendo maltrato y discriminación por el resto de la sociedad; y las acciones para atender estas necesidades se han limitado, por lo general, a construir rampas (la mayoría mal diseñadas) y a establecer tarifas preferenciales en el transporte público, lo cual no las resuelve (ONU-Hábitat, 2015).

La OMS afirma que "sí" podemos darnos el lujo de envejecer si los países, las regiones y las organizaciones internacionales adoptan políticas y programas que mejoren la salud, la autonomía, la productividad y la seguridad de las personas de mayor edad. Esta misma organización ha promovido la idea del "envejecimiento activo"; concepto que significa fomentar políticas que mantengan activo a este sector durante el mayor tiempo posible (Pan, 2017); en este sentido, se han realizado estudios en los cuales se prueba que las personas de la tercera edad activas sufren menos dolencias físicas y emocionales, como la depresión (Pagkaltsos, 2001), y es más probable que sean física y socialmente activas si pueden ir a pasear con seguridad por parques bien iluminados y acceder al transporte local con facilidad (Giró, 2006). 
Por ello, es conveniente propiciar que las personas mayores salgan de sus hogares, que no se excluyan del resto de la sociedad y que no vean el entorno como un obstáculo, sino como una motivación para recuperar su autonomía y dependencia, pues este grupo es la representación del futuro que depara a la mayoría del resto de la sociedad, ya que "nuestra sociedad ha conseguido añadir más años a la vida, pero de momento no es capaz de ofrecer más vida a esos años" (Pérez, 2004).

El objetivo de este artículo es conocer si el diseño del transporte público y la infraestructura urbana actual limitan la movilidad en personas de la tercera edad, contribuyendo a su exclusión social, a fin de proporcionar elementos para renovar los procesos de diseño y evaluación del espacio público como del transporte urbano, explorando las características y las necesidades de las personas de edad avanzada, las dificultades que enfrentan y los requerimientos que tiene para desplazarse por el entorno público mediante la aplicación de una encuesta formulada para responder a esta discusión, la cual se aplicó en Santiago de Querétaro, México. En esta investigación se usó además la metodología cualitativa que produce datos descriptivos y se consideraron como variables la percepción de la infraestructura vial (tiempos de semáforos, estructura del transporte, señalamientos, rampas, guarniciones, accesibilidad), el modo en que operan los transportes (tiempos de parada, accesibilidad de ascenso y descenso, velocidad) y las principales condiciones físicas que presentan personas de edad avanzada.

\section{Envejecimiento y movilidad}

Hoy en día, el criterio para caracterizar a alguien como anciano además de la edad cronológica, que es la edad "oficial", existen otras edades, como la edad social y la psicológica. La edad social se refiere al comportamiento y la edad psicológica alude a los sentimientos. Sin embargo, la única edad estable y mensurable es la cronológica que se reporta a la vida real (Lamprini, 2016).

La vejez es una definición abstracta, considerada como una etapa de la vida y, a nuestro criterio, es un mérito, una distinción o un espacio donde la vida cobra un nuevo sentido (Martínez et al., 2018); para Aguirre y Scavino (2016), es un producto social que se relaciona con otros momentos del ciclo de vida mediante la idea de que ésta ha sido larga y ha posibilitado la acumulación de experiencias. Por ello, la sociedad describe a los adultos mayores de acuerdo con su experiencia, es decir, algunos pueden describir la vejez como etapa feliz y satisfactoria, mientras para otros es una etapa de sufrimiento, enfermedad y padecimientos (Márquez, 2007) en la que se presentan cambios físicos, sociales y psicológicos que pueden provocar limitaciones funcionales, esto quiere decir que el adulto mayor es potencialmente una persona que enfrenta nuevos problemas de interacción con su entorno. 
El acelerado crecimiento de este grupo de población es evidente para cada país, ya que cada vez aumenta la esperanza de vida y se pronostica un mundo envejecido, que, si bien no ocurre de forma homogénea, pues cada país presenta este fenómeno de forma diferente, sí está presente en todo el mundo. La cantidad de población de la tercera edad se triplicó entre 1950 y 2000, pasando de 205.3 a 606.4 millones; en números absolutos, se prevé que para 2030 la población de la tercera edad aumentará a mil 400 millones (OMS, 2016).

Actualmente, una de cada 11 personas tiene más de 65 años; se pronostica que para 2050 sea una de cada seis, esto se señaló en el informe "Perspectivas de la población mundial, 2019". También, para 2018, por primera vez en toda la historia del mundo, las personas de 65 años y más superaron en cantidad a los niños menores de cinco años (ONU, 2019).

En América Latina y el Caribe, la proyección de esperanza de vida será de 81 años para mujeres y 75 años para hombres (OPS, 2015), y se disparará la población de adultos mayores entre 2010 y 2050, y pasará de 59 millones a 196 millones, ya que se prevé que para 2040, habrá 116 personas mayores por cada 100 menores de 15 años en América Latina y 142 personas mayores por cada 100 niños en el Caribe (Huenchuan, 2013).

Figura 1. Porcentaje de la población mundial de 60 años y más respecto a la población total, 1950-2050.

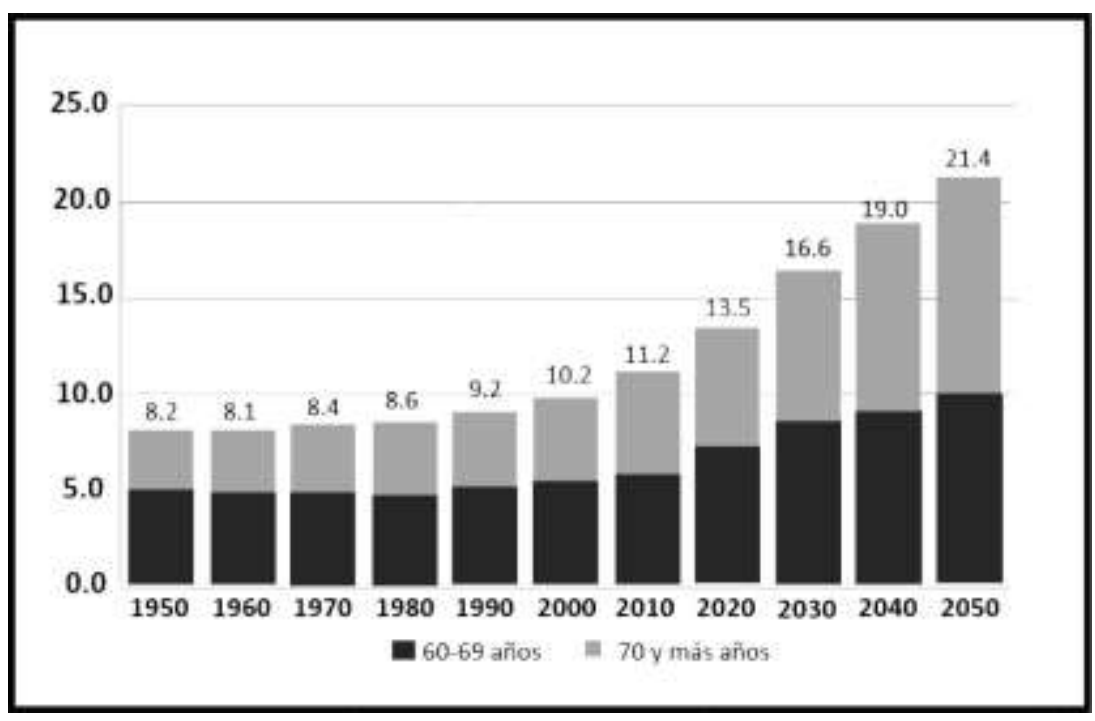

Fuente: Chamie, (2004)

Como el incremento de población longeva aumenta con el paso del tiempo y actualmente su crecimiento es notorio, es importante estudiar las limitaciones que enfrentan este grupo de población, pues entre más aumenta la edad, las condiciones físicas se deterioran como parte del proceso natural de envejecimiento, se dificultan los desplazamientos y la movilidad que, en algún momento de su vida, estas personas estaban acostumbradas a realizar y así forman parte de los grupos sociales vulnerables, con gran impedimento a una participación social, política y económica; al mercado laboral; acceso a 
la educación y otros recursos (Bouskalia, 2003). Para las personas de la tercera edad, el resultado de los cambios en las estructuras sociales (generalmente en todas las sociedades) se refleja en que se sienten perturbados socialmente, ya que se ven obligados a pasar el resto de sus vidas en instituciones, solos o aislados.

Las personas de más de 60 años de edad ven una disminución o hasta una eliminación en las posibilidades de desarrollo y de una vida rica y sana, debido, en mayor parte, a las ideas generalizadas que son consideradas incapaces para las variadas facetas del quehacer humano, siendo excluidas, en su mayoría, de los acontecimientos sociales, dando como resultado un proceso de "invisibilización" (Döring, 2007).

Las personas mayores se identifican como un grupo que enfrentan mayores riesgos de exclusión y se sabe poco sobre las formas en que el envejecimiento y la exclusión se cruzan en el curso de la vida (Scharf et al., 2005). La característica común de la exclusión es la falta de posibilidad del individuo indefenso de ejercer sus derechos debido a las restricciones de acceso que existen en áreas como educación, empleo, vivienda y asistencia médica (Kaftatzoglou, 2006).

Toda persona tiene derecho a un nivel de vida adecuado que le asegure, así como a su familia, la salud y el bienestar, y en especial la alimentación, el vestido, la vivienda, la asistencia médica y los servicios sociales necesarios; tiene asimismo derecho a los seguros en caso de desempleo, enfermedad, invalidez, viudez, vejez u otros casos de pérdida de sus medios de subsistencia por circunstancias independientes de su voluntad Declaración Universal de Derechos Humanos, artículo 25.1 (ONU, 1948).

“Toda persona tiene derecho a la movilidad en condiciones de seguridad vial, accesibilidad, eficiencia, sostenibilidad, calidad, inclusión e igualdad" (Constitución Política de los Estados Unidos Mexicanos, art. 4) (Cámara de Diputados del H. Congreso de la Unión, 1917).

En México los adultos mayores conforman uno de los sectores más desfavorecidos de la población, muy a pesar de la existencia de programas y políticas públicas orientados a mejorar sus condiciones de vida (Osorio, 2016), y es reciente la consideración de la sociedad longeva como categoría social distinta, que, por su edad, tienen derechos y consideraciones especiales. Como resultado al reconocimiento de estos derechos, se advierten los cambios en la Constitución de los Estados Unidos Mexicanos, en la Ley Federal para Prevenir y Eliminar la Discriminación, en el Código Civil de la Federación y de los estados, en la Ley Federal del Trabajo y en la Ley de Asistencia Social (Muñoz-Hernández, 2011), pero también es relativamente reciente la atención de las organizaciones globales en la defensa de las personas de la tercera edad.

Estas acciones y derechos deben atender a las principales necesidades de los ancianos, ya que la exclusión es el producto de la discriminación, el incumplimiento de la ley por parte del Estado y, si se afecta un derecho, se afectan todos; en este sentido, la exclusión social de las personas mayores implica la falta o la negación de recursos, derechos, 
bienes y servicios, a medida que las personas envejecen, así como la incapacidad de participar en las relaciones y actividades normales, disponibles para la mayoría de las personas a lo largo y ancho de la variedad, lo cual conlleva a la afectación de la calidad de vida de las personas mayores, como igualdad y unión de una sociedad que envejece como un todo (Levitas et al., 2007).

Los residentes, en especial los de movilidad restringida, tienen problemas para acceder a los servicios más básicos (Speak y Graham, 2000), por lo que el acceso al transporte público puede ayudarles a aprovechar los bienes, los servicios, los empleos y otras actividades, ya que desempeñará un papel crucial en el mantenimiento de su estilo de vida activo, incluso cuando no puedan conducir (Shrestha et al., 2017).

Facilitar la accesibilidad y movilidad de las personas mayores por la ciudad, incentivando a que salgan de sus hogares, fomentará y prolongará la autonomía e independencia de estos individuos. Así, ellos aprovecharán las oportunidades de crecimiento personal, las cuales surgen de la red social (De Alba, 2017); en este sentido, la movilidad urbana del adulto mayor es "un indicador de autonomía y conservación de la identidad personal, dado que posibilita la realización de otras actividades significativas" (Gajardo et al., 2012).

Las principales necesidades de los ancianos son: ingresos suficientes para cubrir sus necesidades básicas (alimentación, ropa, calzado, limpieza, vivienda, entre otras), interacción social, cobertura médica, actividades (empleo, educación, diversión), acceso a servicios y la capacidad de moverse de forma independiente (Lamprini, 2016); en este sentido, los ancianos hacen uso concurrente de servicios hospitalarios y atenciones médicas, lo cual se traduce en un continuo uso de un medio de transporte para trasladarse a los centros médicos. En México, 34.1\% de las personas de la tercera edad es económicamente activo (INEGI, 2018) y su principal ingreso proviene del trabajo por cuenta propia (CEPAL y OIT, 2018); por tanto, son personas independientes y la movilidad es un aspecto importante para ellos, pues requieren trasladarse a lugares de trabajo. La eficiencia de los servicios es esencial para que la función de la movilidad sea cálida y accesible, es decir, apropiada a las necesidades de las personas de la tercera edad mediante la adaptación y la modificación de la infraestructura y servicios de transporte (Camacho y Araújo-Gomes, 2017).

Además, el movimiento es un mecanismo fundamental en la vida de las personas longevas, pues todos sus sistemas corporales funcionan con mayor eficacia cuando se está activo (OPS, 2002); por ello, diversos organismos internacionales ya se han planteado atender los requerimientos apropiados, en materia de infraestructura, para las personas de edad avanzada y de esta forma hacer frente al fenómeno de envejecimiento (Molina, 2004).

En este sentido, los tres factores que influyen mayormente en la movilidad/accesibilidad de las personas de edad avanzada son: habilidades físicas individuales (como las motrices), aspectos socioeconómicos y culturales (como el ingreso, el 
sector de residencia, el género, el capital social) e infraestructura urbana (como el equipamiento, las condiciones de la viabilidad, la seguridad, el transporte público y privado) (Olivi et al., 2016).

La presencia de espacios públicos, así como los atributos estéticos y paisajísticos del espacio urbano son factores que pueden estimular la movilidad de las personas mayores debido a las oportunidades que ofrecen en términos de interacción social, placer y disfrute estético. Estos aspectos pueden repercutir positivamente sobre la dimensión social e identitaria y sobre las condiciones físicas y de salud de las personas mayores, favoreciendo una mayor satisfacción personal y hábitos de vida más saludables (Olivi et al., 2016). "La calidad de vida de las futuras generaciones de adultos mayores dependerá en buena medida de la capacidad de la sociedad para construir y consolidar las instituciones encargadas de procurar su protección y bienestar" (CONAPO, 2003).

Por otro lado, las investigaciones sobre el estudio del adulto mayor y su movilidad son pocas. Ham y González (2008) analizan, mediante la primera Encuesta Nacional sobre Discriminación en México realizada en 2005, la percepción que la población en general tiene de los adultos mayores, en especial hacia sí mismos; en este estudio, de manera general, la discriminación se define como la situación en la que por prejuicios sobre una persona o grupo se les da un trato desfavorable, en este caso por la edad avanzada, y si han sido discriminados por su condición de viejos, donde ampliamente se comprobó que se sienten discriminados del resto de la sociedad, pues se sienten olvidados.

Por su parte, Davis et al. (2011) describen tanto la frecuencia como el propósito y el modo de los viajes cotidianos de las personas mayores de 70 años, tomando los datos como actividades físicas y asociándolos con condiciones de mejoras de salud. La investigación se realizó mediante la selección de un grupo de 214 personas de 70 años y más, a quien se le entregó un Actigraph (pequeño dispositivo electrónico que sirve para monitorear y registrar cierta actividad de una persona en un determinado periodo), a fin de registrar los viajes efectuados fuera y dentro de la casa, así como el propósito del viaje (compras, visitas, negocios, paseos, entretenimiento, salir a comer, entre otras) y también el modo de transporte, durante nueve días. Los resultados arrojaron que las personas con menos viajes registrados fueron aquellas con mayor edad, con baja función física (ayuda para caminar), con educación baja, que vivían en áreas más desfavorables y con solo uno o ningún automóvil en casa.

Copeland y Esliger (2009) también estudian la actividad física de personas de edad adulta, pues el porcentaje de inactividad ha aumentado drásticamente los últimos años. El análisis se presenta en un grupo de adultos mayores sanos y por medio de un acelerómetro evalúan la actividad física de grupo durante siete días; se revela que los episodios más largos de actividad de los adultos mayores ocurren en la mañana en un intervalo de 6 am a 12 pm, pero que son de baja intensidad, ya que conforme la edad va aumentando en una persona tarda más tiempo en realizar ciertas actividades. 
Por otro lado, Döring (2007) entrevista un grupo de adultos mayores, el cual afirma la falta de puntos de reunión adecuados o no exclusivos para facilitar la socialización de personas longevas, pues, aunque la economía sea estable para algunos, se siguen enfrentando dificultades para este tipo de encuentros, pues casi todas las oportunidades parecen estar diseñadas para quienes no han alcanzado la cuarentena de años. "Fomentar la movilidad urbana de las personas mayores es una inversión social, que impacta directamente en la autonomía e independencia de este, cada vez más creciente, grupo de la población" (Laboratorio de Innovación Pública, 2018).

\section{Metodología}

Se trata de un estudio cualitativo exploratorio mediante la aplicación de encuestas domiciliadas y exploratorias a personas de la tercera edad, el cual se detalla a continuación.

\section{Cuestionario}

El cuestionario se diseñó con base a la Encuesta Nacional de Movilidad y Transporte propuesto por la UNAM (2015) y adaptado para dar resultados a nuestro estudio, acudiendo al recuerdo pre-Covid y a la actualidad; consta de siete apartados:

a) Uso de medios de transporte por nuestra población en estudio en la que se hacen preguntas puntuales, como el transporte más usual, ya sea privado o público, y aspectos del medio de transporte.

b) Número de viajes que realizan a la semana, horario preferido y acompañantes, entre otras preguntas.

c) Desplazamientos a pie (caminatas), qué tanto les gusta caminar, propósitos de las caminatas, duración de éstas.

d) Percepción del transporte y de la infraestructura urbana.

e) Condiciones físicas que presentan los encuestados, como enfermedades y discapacidades y su relación con el uso del transporte público y la infraestructura urbana.

f) Mejoras que les gustarían que se le realizaran al transporte público y a la infraestructura urbana.

g) Tres preguntas abiertas personales referentes a la percepción de exclusión, las cuales son opcionales de contestar o no.

Este cuestionario fue entregado junto con una carta de consentimiento informado (asegurando la confidencialidad y el anonimato), y fue aplicado de manera domiciliada para incluir a todas las personas longevas y no sólo a aquellas que hacen uso del transporte público o de la infraestructura urbana, y así no alterar el objetivo del estudio y que fuera de forma exploratoria, ya que no se conocía con exactitud la ubicación donde habitan personas 
de la tercera edad (en el hogar seleccionado, se preguntaba si habitaba alguien de este sector; al ser confirmado, se procedía a la entrega o aplicación de la encuesta y al registro de la vivienda; al ser negativa la respuesta, se buscaba otro hogar). Se puede consultar el formato de la encuesta en la información de los autores y complementando aquellos datos con los obtenidos de una pequeña entrevista realizada en una reunión de adultos mayores el día 1 de octubre del 2019.

\section{Participantes}

Las encuestas fueron aplicadas a personas de la tercera edad, es decir de 60 años o más, que habitaban en el hogar seleccionado aleatoriamente (solo una por hogar).

\section{Tamaño de la muestra}

El tamaño de la muestra se llevó a cabo por medio de 4 pasos:

a) Se concentraron todas las Áreas geo estadísticas básicas urbanas (AGEB'S URBANAS) pertenecientes a la cabecera municipal de Santiago de Querétaro.

b) El tamaño de la muestra se calculó por medio de poblaciones finitas, pues así es la población, es decir conocemos el total de la población y deseamos saber cuántos del total tendremos que estudiar. De los datos proporcionados por INEGI (Encuesta intercensal 2015), se obtiene la población total correspondiente a Santiago de Querétaro: 648,674 personas; de esta misma base de datos, mediante un filtrado, se obtiene el número de habitantes de la tercera edad, el cual es de 73,040 personas, esto es $11.26 \%$. El total de la muestra es de 75 observaciones a realizar de forma exploratoria y domiciliada en los diferentes AGEB'S.

c) Se seleccionaron de forma aleatoria sistemática 75 AGEB'S de las 241 correspondientes a Santiago de Querétaro. Como ciertas AGEB'S cuentan con un número de manzanas mayor que otras, se sacaron pesos proporcionales.

d) Se enumeró cada grupo de manzanas perteneciente a cada AGEB seleccionado; de forma aleatoria, se eligieron tres opciones de manzanas para la aplicación de encuestas, por si se presentaran inconvenientes, como la falta de personas de la tercera edad, inseguridad, negación de proporcionar información, entre otras. 


\section{Análisis de datos}

Con los datos recabados por las encuestas, se procedió a la verificación de resultados, eliminando aquellos que no se consideraron correctos debido a la mala captura de datos o a la mala coherencia del encuestado; se remplazaron aplicando las encuestas nuevamente hasta alcanzar el tamaño real de la muestra (75 encuestas); después, se capturaron los datos en una base de codificación en Excel y se realizó un análisis estadístico de los datos, acudiendo a la estadística descriptiva, la cual permite caracterizarlos y obtener conjuntos representativos con las variables más significativas.

\section{Resultados}

Se presenta la estadística descriptiva de los datos y los resultados obtenidos del análisis de las variables evaluadas durante el estudio sobre la opinión que tienen las personas de la tercera edad acerca del transporte público y la infraestructura urbana mediante 12 secciones, las cuales se describen a continuación.

Tabla 1. Evaluación de los aspectos del Camión/Qrobús

\begin{tabular}{|c|c|c|c|c|c|c|c|c|c|c|c|c|c|}
\hline & $\frac{\frac{\pi}{x}}{\frac{5}{3}}$ & $\frac{\vec{g}}{\stackrel{\vec{n}}{*}}$ & 胥 & 亳 & $\frac{\mathrm{E}}{\mathrm{E}}$ & $\frac{\theta}{b}$ & $\frac{\mathfrak{g}}{\frac{\tilde{E}}{\mathrm{E}}}$ & $\frac{\mathscr{E}}{\stackrel{\Xi}{\tilde{Z}}}$ & 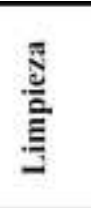 & $\frac{\frac{\mathrm{g}}{\mathrm{E}}}{\frac{\mathrm{E}}{\mathrm{n}}}$ & 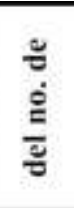 & 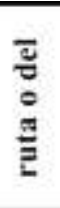 & 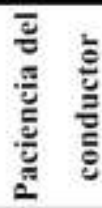 \\
\hline Promedio & 4.80 & 4.60 & 4.20 & 2.20 & 3.20 & 2.40 & 5.00 & 4.20 & 5.00 & & 3.67 & & 3.60 \\
\hline
\end{tabular}

Fuente: elaboración propia.

La tabla 1 muestra la evaluación de los aspectos del medio de transporte Camión/Qrobús (principal medio de transporte público en Santiago de Querétaro) en una escala de 0 a 5 , donde 0 es pésimo y 5 excelente. Se observa la ineficiencia de este medio de transporte en cinco principales aspectos: tiempo de espera, costo, cobertura (por lo general deben tomar dos rutas o caminar una gran distancia para llegar al destino), paciencia del conductor para que puedan abordar o descender de la unidad y visibilidad para identificar el número de ruta.

La tabla 2 indica la opinión acerca del mejor transporte de su localidad o ciudad, donde las tres principales respuestas fueron: automóvil particular, taxi y camión/Qrobús; un porcentaje pequeño prefiere los diferentes medios de transporte restantes: colectivo/combi, autobús foráneo, Uber/Didi/Cabify, motocicleta, bicicleta, entre otros. 
Tabla 2. Mejor transporte de la localidad

\begin{tabular}{lcccc}
\hline & Camión/Qrobús & Taxi & $\begin{array}{c}\text { Automóvil } \\
\text { particular }\end{array}$ & Otro \\
\hline Promedio & $26.0 \%$ & $28.0 \%$ & $39.0 \%$ & $7.00^{\circ} \%$ \\
\hline
\end{tabular}

Fuente: elaboración propia.

En la tabla 3 se observa el promedio del estado de conservación de algunos elementos de la infraestructura urbana; para ello, se utiliza la misma escala que en la tabla 1 ( 0 a 5, es decir, de pésimo a excelente). Los puentes peatonales, en su mayoría, son muy inclinados y con dimensiones altas, por lo que optan por tener que caminar hasta el siguiente cruce peatonal; por otro lado, tienen que esperar el medio de transporte un tiempo considerable, pero la mayoría de veces no hay donde sentarse, por lo que esperar el transporte, por más de 30 minutos, se torna cansado. La evaluación de las ciclovías no se tomó en cuenta, pues casi todas las personas estudiadas preferían no evaluarlas, ya que, al no hacer uso de ellas, ignoran su estado; por consiguiente, se deduce que la bicicleta es de los medios de transporte menos utilizados por la población longeva en Santiago de Querétaro.

Tabla 3. Estado de conservación de la infraestructura urbana

\begin{tabular}{|c|c|c|c|c|c|c|c|c|c|c|}
\hline & 总 & 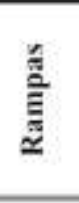 & 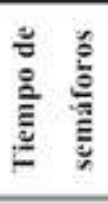 & 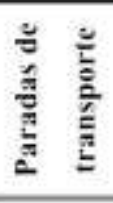 & 总 & 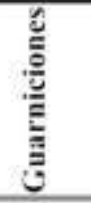 & $\frac{\stackrel{y}{z}}{\hat{E}}$ & 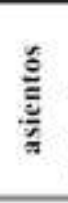 & 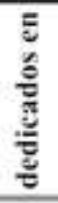 & $\frac{\frac{\pi}{3}}{\frac{6}{2}}$ \\
\hline Promedio & +14 & 3.67 & 4,00 & 1.83 & 1.71 & 2.57 & & 4,00 & & - \\
\hline
\end{tabular}

Fuente: elaboración propia.

La mayoría de la población considera que el transporte público es seguro y cómodo, pero aspectos como la eficiencia, la rapidez y el costo aún son considerados inadecuados (ver tabla 4).

Tabla 4. Consideración del transporte público

\begin{tabular}{cccccc}
\hline & Eficiente & Rápido & Barato & Seguro & Cómodo \\
& & & & & \\
\hline SI & $15.0 \%$ & $28.6 \%$ & $14.3 \%$ & $85.7 \%$ & $71.0 \%$ \\
NO & $85.0 \%$ & $71.4 \%$ & $85.7 \%$ & $14.3 \%$ & $29.0 \%$ \\
\hline
\end{tabular}

Fuente: elaboración propia. 
Entre las principales razones expresadas por los encuestados para no utilizar el transporte público son: costo elevado, dificultad para abordarlo, descenderlo y transitar dentro de éste mismo, así como que un número alto dispone de un automóvil particular (ver tabla 5).

Tabla 5. Principales razones por las que no usan transporte público

\begin{tabular}{lc}
\hline $\mathbf{1}$ & Es muy caro \\
$\mathbf{2}$ & Es incómodo \\
$\mathbf{3}$ & Tengo carro \\
\hline
\end{tabular}

Fuente: elaboración propia.

Por otro lado, un número alto de los encuestados padece enfermedades o discapacidades, como diabetes, hipertensión y depresión, pero no son impedimento para usar el transporte público; sin embargo, un porcentaje bajo de personas presenta dolencias en las rodillas y/o cadera y ceguera, por lo que se enfrenta a problemas con las paradas de transporte, ya que deben esperar un tiempo elevado de pie para su abordaje, así como la escasez de áreas reservadas o, incluso, la falta de respeto del resto de la sociedad por estos lugares designados a la exclusividad del adulto mayor (ver tabla 6).

Tabla 6. Problemas para usar el transporte público debido a alguna enfermedad o discapacidad

\begin{tabular}{|c|c|c|c|c|c|}
\hline & Acceso & $\begin{array}{l}\text { Falta de } \\
\text { áreas } \\
\text { reservadas }\end{array}$ & $\begin{array}{l}\text { Infraestructura } \\
\text { especial en el TP }\end{array}$ & $\begin{array}{c}\text { Infraestructura } \\
\text { especial en las } \\
\text { paradas }\end{array}$ & Ninguna \\
\hline Promedio & $28.6 \%$ & $9.4 \%$ & $11.7 \%$ & $7.1 \%$ & $43.2 \%$ \\
\hline
\end{tabular}

Fuente: elaboración propia.

Se preguntó a la población estudiada sobre su participación en alguna actividad social, como deportiva, cultural, religiosa, y sobre si el transporte público mejorara, podrían intervenir en más acciones; sin embargo, prefieren seguir desarrollando sus actividades cotidianas (tabla 7). Es importante continuar motivando a este sector de la población a realizar más actividades para que lleven una vida activa. 
Tabla 7. Realización de actividades sociales con la mejora del transporte público

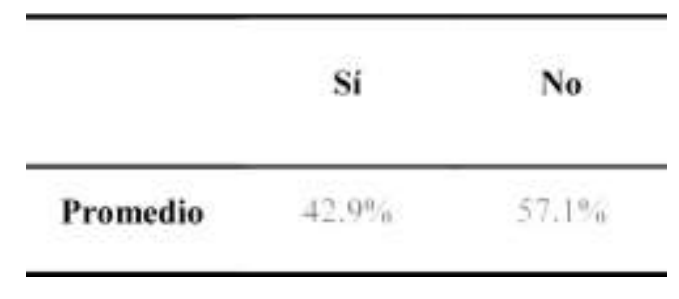

Fuente: elaboración propia.

Las tablas 8 y 9 muestran las principales mejoras que la sociedad envejecida estudiada preferiría que se tomaran en cuenta para su movimiento libre e independiente; en cuestiones del transporte público, las mejoras más mencionadas fueron acerca de la paciencia del chofer para que puedan abordar o descender del transporte público; mencionan sentirse presionados y con ello ser torpes, lo cual, muchas veces, conlleva a golpes e, incluso, caídas; también aluden a la falta de paciencia de esperar a que tomen un lugar, ya que en cuanto suben a la unidad de inmediato se arranca.

Otros aspectos señalados fueron acerca del tiempo de espera, ya que se torna cansado y desgastante esperar por un tiempo significante a que llegue el medio de transporte, y del costo, pues el gobierno debe tener en cuenta que muchos de ellos ya no son económicamente activos. Por otro lado, en cuestión de infraestructura urbana, a la mayoría les gustaría que se pusiera más atención en las guarniciones (banquetas), pues no pueden ser transitadas o no hay continuidad debido a su deterioro, mala construcción, desnivel o, incluso, a su ausencia, por lo que los adultos mayores suelen tropezarse o tienen que bajarse de ellas y más adelante volver a subirse, pues en ocasiones se encuentran obstaculizadas por basura, puestos o el desquebraje por las raíces de los árboles.

También se coincide en los cruces peatonales, ya que el tiempo para cruzar es muy corto y las longitudes muy largas, asimismo, los puentes peatonales les resulta imposible subirlos.

Finalmente, se capturaron comentarios de la población estudiada acerca del mal sistema de drenaje o alcantarillado de las localidades, pues al caminar en tiempos de lluvia, ya sea por recreación, ejercicio o adquirir un bien o servicio en proximidad, enfrentan un reto para no mojarse los pies o para no resbalarse a causa del agua estancada; situación que los lleva a dolencias reumáticas.

Tabla 8. Mejoras del transporte público

\begin{tabular}{cc}
\hline $\mathbf{1}$ & Paciencia de los choferes \\
$\mathbf{2}$ & Disminuir tiempo de espera \\
$\mathbf{3}$ & Costo \\
\hline
\end{tabular}

Fuente: elaboración propia. 
Tabla 9. Mejoras de la infraestructura urbana

\begin{tabular}{lc}
\hline $\mathbf{1}$ & Guarniciones \\
$\mathbf{2}$ & Cruces Peatonales \\
$\mathbf{3}$ & Alcantarillas \\
\hline
\end{tabular}

Fuente: elaboración propia.

La tabla 10 confirma que un amplio número de las personas de la tercera edad se ha sentido o se siente excluido del resto de la sociedad por su condición de viejo; las razones son numerosas, pero entre las más mencionadas destacan (ver tabla 11): la mayoría de las veces los choferes prefieren no hacerles la parada, al menos que se encuentre un grupo de personas más jóvenes ("para no perder pasaje"); al mismo tiempo prefieren salir en horarios cuando el resto de la sociedad esté trabajando o realizando sus actividades, ya que muchas veces pierden la sensibilidad hacia ellos y se les olvida que su condición es más lenta al subir escaleras, caminar, cruzar calles e, incluso, al cederles el asiento.

Tabla 10. Exclusión social

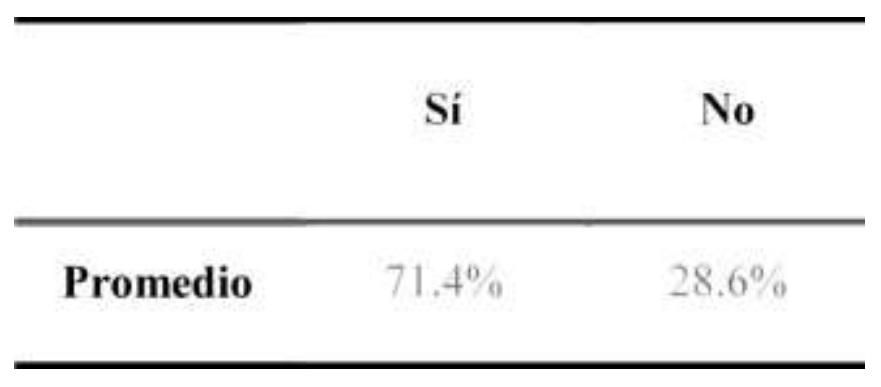

Fuente: elaboración propia.

Tabla 11. Principales razones de exclusión

\begin{tabular}{lr}
\hline $\mathbf{1}$ & Choferes no les hacen la parada \\
$\mathbf{2}$ & La gente pierde sensibilidad \\
$\mathbf{3}$ & No siempre ceden los asientos reservados \\
\hline
\end{tabular}

Fuente: elaboración propia. 
Tabla 12. Confianza al salir si el transporte mejorara

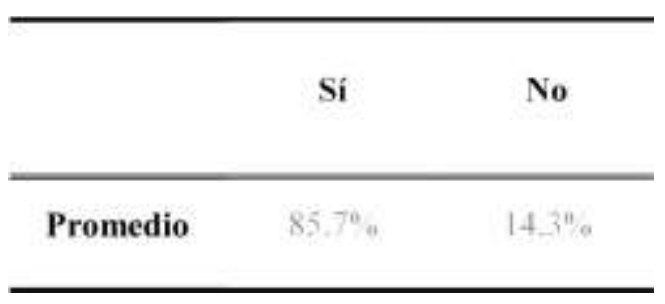

Fuente: elaboración propia.

Por último, en la tabla 12 se aprecia la gran diferencia entre la respuesta positiva y la negativa acerca de plantear hipotéticamente la mejora del transporte público y la infraestructura urbana atendiendo las demandas mencionadas y, como consecuencia, la mayor confianza que las personas de la tercera edad tendrían para salir y desplazarse con libertad al lugar que ellos deseen. Durante la aplicación de la encuesta y con autorización previa, se muestran algunos relatos de experiencias reales contadas por algunas personas de la tercera edad entrevistadas:

\footnotetext{
"De manera muy difícil uno puede subir y bajar los escalones del camión, ya que son un tanto elevados, y me siento presionada por parte de los demás usuarios y el chofer para hacerlo de la manera más rápida posible lo que ocasionalmente provoca que me golpee” Guadalupe Guerrero.

“El tiempo de espera se torna cansado y más si no hay un lugar en donde esperar sentado” Román Escamilla.

"Prefiero salir cuando sé que la gente ya está ocupada en otras actividades, ya que si se acumula bastante gente éstos pierden sensibilidad por nosotros, ya que su prioridad es sólo abordar sin importarles nadie pues se les hace tarde" - Señora Ignacia.

"Me es difícil reconocer el camión al que debo subir, ya que casi no veo bien el letrero de destino y a veces me doy cuenta ya que pasó y así hasta como 3 veces" Mariano Salgado.
}

\section{Discusiones}

Al no ser adecuado el diseño del transporte público para el uso de personas de la tercera edad, contribuye a la exclusión social y fomenta que este grupo prefiera no salir de sus hogares para no batallar con las dificultades y con la infraestructura urbana, como las expresadas anteriormente por algunos entrevistados. Con ello, este estudio proporciona elementos básicos para atender el efecto de exclusión del entorno hacia el adulto mayor, como la innovación en el diseño del transporte público y la infraestructura urbana, así como capacitaciones de sensibilidad a choferes, ya que, en la actualidad, como lo menciona ONUHábitat (2015), la resolución para este problema se ha resumido en proporcionar tarifas preferenciales y en colocar rampas, generalmente mal diseñadas. 
Es importante mencionar que las enfermedades más comunes en esta etapa de la vida no son las principales causas de limitación al uso de medios de transporte público e infraestructura urbana; sin embargo, la dolencia de articulaciones, también común en la vejez, sí ha llegado a impedir que los adultos mayores opten por no salir al entorno y esto conlleva al aislamiento, el cual puede ocasionar depresión, padecimiento usual en esta etapa; por ello, esta investigación coincide con Giro (2006) respecto a hacer lo posible para que la vida de los ancianos sea más activa y estén motivados a salir, sin presentar al transporte público y a la infraestructura urbana como un pesar o un problema, pues se demostró que el número de ancianos participantes en alguna actividad social aumentaría sí el transporte y la infraestructura tuvieran mejoras.

Por otro lado, el medio menos utilizado por parte de este grupo de personas es la bicicleta, ya que sus condiciones físicas imposibilitan su uso para trasladarse, por lo que, para fines de esta investigación, es el único transporte que se descarta, y no por las condiciones que presenta el transporte como tal.

Ham y González (2008), analizaron la percepción que la población tenía sobre los adultos mayores, así como la percepción de ellos mismos y confirmaron el sentimiento de discriminación/exclusión por el resto de la sociedad y, aunque ha pasado más de una década, es evidente que no se ha presentado la atención necesaria para esta situación, ya que esta investigación recopila que, en la actualidad, más de la mitad de los encuestados se sienten excluidos por su condición de viejos.

Sin embargo, aunque se han realizado este tipo de investigaciones, la revisión de la literatura afirma que la temática está poco estudiada, principalmente en México; por ello, es importante abordar con seriedad el problema aquí desarrollado con el fin de promover su solución. Se recomienda hacer nuevas investigaciones sin acudir al recuerdo (por la actual contingencia se tuvo que limitar a esta modalidad) y en un entorno donde el transporte público sea más amplio y con mayor frecuencia, ya que el lugar de estudio del presente (Santiago de Querétaro) aún no cuenta con muchas opciones de transporte público y unidades disponibles, lo que limita la frecuencia, que es uno de los principales problemas señalados por la población analizada, y con un mayor tamaño muestral, pues también por la actual contingencia el valor del nivel de confianza se disminuyó y el de precisión se aumentó.

\section{Conclusiones}

Este artículo ha querido mostrar la importancia de estudiar a los adultos mayores y su comportamiento de autoexclusión del resto de la sociedad, ya que suelen aislarse en sus hogares; asimismo, es imperante saber si es posible hacerle frente, pues este grupo de personas conforma una cifra alta del total de la población y su aumento acelerado es evidente; además, es la etapa de la vida que depara a la mayoría y que el movilizarse de forma independiente para satisfacer gustos y necesidades es un derecho de cada individuo; 
por tanto, el trasporte público y la infraestructura urbana no se ha diseñado para todos, incluso para aquellos con capacidades más limitadas en comparación a los demás, lo cual restringe dicho derecho.

La revisión bibliográfica indica una escasez de artículos donde se mencionan mejoras o adaptaciones en el transporte público ante las necesidades de las personas de la tercera edad e, incluso, sobre su movilidad; aunque se hayan firmado convenios y tratados o realizado asambleas y conferencias como las que se recopilaron y citaron anteriormente, es visible que no hacen una diferencia notable, pues hasta la fecha este sector sigue siendo un grupo vulnerable.

Por ello, se requiere prestar atención a la adaptación del entorno a fin de asegurar una vejez plena sin limitaciones de accesibilidad a servicios básicos e, incluso, a actividades de ocio, enfocándose principalmente en la movilidad urbana que garantice la seguridad y la adaptabilidad para que las personas longevas puedan hacer uso de cualquier medio y modo de transporte en el momento que lo requieran o prefieran y así fomentar y fortalecer su independencia sin importar su edad, logrando una vida activa y sana; además, es necesario enmarcar que apoyos como descuentos en pasajes y una cantidad escasa de asientos reservados en el transporte público, como en el caso de México, no son medidas suficientes para que el adulto mayor se sienta cómodo de usarlos.

Por tanto, al tratar de reconocer si el diseño del transporte público y de la infraestructura urbana como está en la actualidad contribuyen a la exclusión social de las personas longevas, los resultados encontrados concluyen que mayormente sí se han sentido excluidos, sobre todo porque los conductores no les hacen la parada al menos que haya más gente para abordar la unidad, lo cual también ocasiona problemas, ya que éstos pierden sensibilidad por ellos y se les olvida que sus condiciones son más lentas y torpes; asimismo, otra causa de conflicto son aspectos de la infraestructura urbana, como las banquetas, pues en esta etapa de la vida las personas habitualmente arrastran los pies y casi todas las banquetas estén deterioradas, lo cual ocasiona que tropiecen, por lo que prefieren salir en automóvil particular o con algún acompañante para sentirse con mayor confianza y en un horario de media mañana para no tener tanto contacto con la sociedad que trabaja.

Para reafirmar este hecho, la población longeva encuestada afirma que participaría en alguna actividad social si es que el entorno llegase a mejorar a su favor para tener más confianza al salir. Exhortamos a las personas longevas a salir y no al aislamiento, ya que como ellos lo señalan "es mejor quedarse en casa, cuando ya sólo representas una carga para la sociedad"; por ello, es importante impulsar a la población en general a incluir la participación de los adultos mayores, no con la perspectiva de un gasto a la modificación del entorno, sino como una inversión futura para una sociedad sana que cada vez envejece. 


\section{Referencias}

Aguirre Cuns, R. y Scavino Solari, S. (2016). Cuidar en la vejez: desigualdades de género en Uruguay. Papeles del CEIC, International Journal on Collective Identity Research, 2016 (1), 1-41. Recuperado de https:/ / dialnet.unirioja.es/servlet/articulo?codigo $=5416621$

Bouskalia. (2003). Atoma Disabled. Atenas: Gull.

Camacho, A. y Araújo-Gomes, F. (2017). Elderly and urban mobility: a reflective approach to nursing. Journal of Nursing, 11 (12), 5066-5073. doi: http:/ / dx.doi.org/10.5205/1981-8963-v11i12a23068p5066-5073-2017

Cámara de Diputados del H. Congreso de la Unión (1917). Constitución Política de los Estados Unidos Mexicanos. Art. 4. Párrafo adicionado DOF 08-05-2020, México.

CEPAL y OIT. (2018). Coyuntura Laboral en América Latina y el Caribe. La inserción laboral de las personas mayores: necesidades y opciones. Recuperado de: https://repositorio.cepal.org/handle/11362/43603

Chamie, J. (2004). World Population Prospects The 2002 Revision. Nueva York: United Nations.

CONAPO (2003). Índices de desarrollo social en las etapas del curso de vida. México: Consejo Nacional de Población.

Copeland, J., L. y Esliger, D., W. (2009). Accelerometer assessment of physical activity in active, healthy older adults. Journal of aging and physical activity. 17 (1), 17-30. doi https://doi.org/10.1123/japa.17.1.17

De Alba, M. (2017). Representaciones sociales y experiencias de vida cotidiana de los ancianos en la Ciudad de México. Estudios demográficos y urbanos. 32 (1), 9-36. Recuperado de http:/ / www.scielo.org.mx/scielo.php?script=sci_arttextypid=S0186$72102017000100009 y \operatorname{lng}=$ esynrm=iso

Davis, M., G., Fox, K., R., Hillsdon, M., Coulson, J., Sharp, D., J., Stathi, A., y Thompson, J., L. (2001). Getting out and about in older adults: the nature of daily trips and their association with objectively assessed physical activity. International Journal of Behavioral Nutrition and Physical Activity. 8 (116), doi: https://doi.org/10.1186/1479-5868-8-116

Dörign, M. (2007). Un nuevo enfoque de la vejez. Iztapalapa, Revista de Ciencias Sociales y Humanidades, (62), 193210 Recuperado de https://revistaiztapalapa.izt.uam.mx/index.php/izt/article/view/327

Gajardo, J., Navarrete, E., López, C., Rodríguez, J., Rojas, A., Troncoso, S., y Rojas, A. (2012). Percepciones de personas mayores sobre su desempeño en el uso de transporte público en Santiago de Chile. Revista Chilena de Terapia Ocupacional. $12 \quad$ (1), 88-102. Recuperado de https://revistas.uchile.cl/index.php/RTO/article/view/22055

Giró, J. (2006). El envejecimiento activo en la sociedad española. Envejecimiento activo, envejecimiento en positivo, España: Universidad de la Rioja,

Ham , R. y González , C. (2008). Discriminación en las edades avanzadas en México. Papeles de población. 14 (55), 35-58 Recuperado de http://www.scielo.org.mx/scielo.php?script=sci_arttextypid=S1405$74252008000100003 y \operatorname{lng}=$ esynrm=iso

Huenchuan, S. (2013). Envejecimiento, solidaridad y protección social en América Latina y el Caribe: la hora de avanzar hacia la igualdad. Chile; Comisión Económica para América Latina y el Caribe (CEPAL).

INEGI (2015). Encuesta Intercensal. Instituto Nacional de Estadística y Geografía, México. Recuperado de https://www.inegi.org.mx/programas/intercensal/2015/

INEGI. (2018). Encuesta Nacional de Ocupación y Empleo (ENOE). México. Recuperado de https://www.inegi.org.mx/programas/enoe/14ymas/

Kaftatzoglou (2006). Social exclusion: inside, outside and under theoretical, historical and political origins of an ambiguous concept. Atenas: Savalas.

Laboratorio de Innovación Pública. (2018). Fomentando la movilidad de los adultos mayores en la ciudad. Santiago de Chile: RedActiva. Recuperado de https://www.fiapinternacional.org/redactivafomentando-la-movilidad-de-los-adultos-mayores-en-la-ciudad-laboratorio-de-innovacion-publicalip-marzo-2018/

Lamprini, K. (2016). Exclusión social de los ancianos. J Healthc Commun. 1 (3), Recuperado de https:// healthcarecommunications.imedpub.com/social-exclusion-of-elderly.php?aid=9866 
Levitas, R., Pantazis, C., Fahmy, E., Gordon, D., Lloyd-Reichling E. y Patsios, D. (2007). The multi-dimensional analysis of social exclusion. Londres: Universidad de bristol.

Márquez, T. (2007). Transición: envejecer no es de cobardes. Estado de México. México: Norma.

Martínez, T.J., González Aragón, C.M., Castellón Léon, G. y González Aguiar, B. (2018). El envejecimiento, la vejez y la calidad de vida: ¿éxito o dificultad?. Revista Finlay, (8), 59-65, Recuperado de http://scielo.sld.cu/scielo.php?script=sci_arttextypid=S2221-24342018000100007ylng=esytlng=es.

Martínez Sellarés, R. (2012), Proceso de envejecer, Enfermería y evenjecimiento. España: Masson.

Molina, V. (2004). Aproximaciones al espacio urbano de la vejez en el Distrito Federal. México: Anuario de Espacios Urbanos.

Morfi Samper, R. (2010). Gestión del cuidado en Enfermería. Revista Cubana de Enfermería. 26 (1), 1-2 Recuperado de $\quad$ http://scielo.sld.cu/scielo.php?script=sci_arttextypid=S0864$03192010000100001 \mathrm{ylng}=$ esynrm $=$ iso $>$.

Muñoz-Hernández, R. (2011). Las políticas públicas de la vejez en México 2010. Iztapalapa, Revista de Ciencias Sociales $y$ Humanidades. (71), 35- Recuperado de https:// www.redalyc.org/articulo.oa?id=393/39348325003

Olivi, A., Fadda, G. y Reyes, V. (2016). Movilidad urbana y calidad de vida de las personas mayores en una ciudad vertical. El caso de Valparaíso, Chile. Revista Márgenes. 13 (19), 38-47. Recuperado de https://revistas.uv.cl/index.php/margenes/article/view/1033

OMS (2016). Acción multisectorial para un envejecimiento sano basado en el ciclo de vida: proyecto de estrategia y plan de acción mundiales sobre el envejecimiento y la salud. Organización Mundial de la Salud. Recuperado de https://apps.who.int/gb/ebwha/pdf_files/WHA69/_A69_17-sp.pdf

ONU-Hábitat (2015). Reporte nacional de movilidad urbana en México 2014-2015. México. Recuperado de http://conurbamx.com/home/wp-content/uploads/2015/07/Reporte-Nacional-de-MovilidadUrbana-en-Mexico-2014-2015-Final.pdf

ONU (1948). Declaración Universal de Derechos Humanos. Recuperado de: https://www.un.org/es/aboutus/universal-declaration-of-human-rights

ONU (2019). Informe de la ONUV 2019. Organización De Las Naciones Unidas Recuperado de https://population.un.org/wpp/Publications/Files/WPP2019_PressRelease_ES.pdf

ONU (2019). Perspectivas de la Población Mundial 2019. Organización de las Naciones Unidas. Recuperado de https://population.un.org/wpp/

OPS (2002). Evaluación funcional del adulto mayor. Guía clínica para atención primaria a las personas adultas mayores. Organización panamericana de la salud.

OPS (2015). Estrategia y plan de acción sobre demencias en las personas mayores. Organización Panamericana de la Salud

Recuperado de https://www.paho.org/hq/index.php?option=com_docmanytask=doc_downloadygid=31930yIte mid=270ylang=es.

Osorio, Ó. (2016). Envejecimiento Poblacional: discriminación y políticas públicas integrales. Iztapalapa, Revista de Ciencias Sociales y Humanidades. (81), 141-172 Recuperado de https://revistaiztapalapa.izt.uam.mx/index.php/izt/article/view/57/93

Osorio, P. (2006). Exclusión Generacional: La Tercera Edad. Revista Mad. Revista del Magíster en Análisis Sistémico

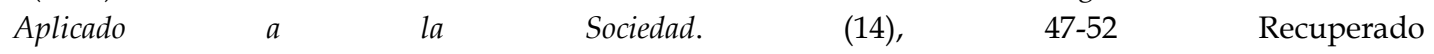
de https://www.redalyc.org/articulo.oa?id=3112/311224740006.

Pan, L-T. (2017). Vejez y envejecimiento en china. Estudios de Asia y África. (52), 459-470 Recuperado de https://doi.org/10.24201/eaa.v52i2.2322

Pagkaltsos (2001). Gerontology and Geriatrics elements. Grecia: Technological Educational Institute of Thessaloniki Pérez, P. (2004). ¿Por qué envejecemos? Cómo añadir años a la vida y vida a los años. Madrid: Eneida.

Scharf, T., Phillipson, C. y Smith, A. (2005). Social exclusion of older people in deprived urban communities of England. European Journal of Ageing. 2(). 76-87. doi: https://doi.org/10.1007/s10433-005-0025-6 
Shrestha, B., Millonig, A., Hounsell, N. y Mcdonald, M. (2017). Revisión de las necesidades de transporte público de las personas mayores en el contexto europeo. Revista de envejecimiento de la población. 10 (4), 343-361. doi https:/ / doi.org/10.1007/s12062-016-9168-9

Speak, S. y Graham, S. (2000). Private sector service withdrawal in disadvantaged neighbourhoods. Gran Bretaña: Policy Press.

UNAM (2015). Encuesta Nacional de movilidad y Transporte. Universidad Nacional Autónoma de México. México. Recuperado de http://www.losmexicanos.unam.mx/movilidadytransporte/encuesta_nacional.html

Zamorano, C., Alba, M., Capron, G. y González, S. (2012). Ser viejo en una metrópoli segregada: adultos mayores en la ciudad de México. Nueva antropología. 25 (76), 83-102 Recuperado de http:/ / www.scielo.org.mx/scielo.php?script=sci_arttextypid=S0185-

06362012000100005ylng=esytlng=es. 